\title{
Die Ursachen für die bessere Anpassungsfähigkeit der Polyploiden
}

\author{
Von Georg Melchers \\ Aus dem Kaiser-Wilhelm-Institut für Biologie, Tübingen \\ (Z. Naturforschg. 1, 160-165 [1946]; eingegangen am 13. März 1946)
}

\begin{abstract}
Aus pflanzengeographischen Untersuchungen und der Tatsache, daß fast alle bedeutenden Kulturpflanzen Polyploide sind, muß auf ihre größere Anpassungsfähigkeit* geschlossen werden. Die experimentell frisch hergestellten Polyploiden geben keine direkten Anhaltspunkte für die Erklärung dieser Erscheinung. Die hier entwickelte und durch einige Beispiele erläuterte genphysiologische Vorstellung macht die bessere Anpassungsfähigkeit der Polyploiden auch bei Berücksichtigung der Tatsache, daß frisch hergestellte Polyploide eine Verbesserung nicht sofort erkennen lassen, verständlich: In Polyploiden lassen sich Gene für selektionswürdige Merkmale in größerer Menge anreichern als in Diploiden. Die Wirkung mancher Gene steigt auch über die zweifache Quantität hinaus noch an. Das erklärt die größere Anpassungsfähigkeit der Polyploiden. Aber nicht jedes Genom enthält von vornherein selektionswürdige Merkmale fördernd beeinflussende Gene, deren Wirkung sich über die zweifache Quantität hinaus steigern läßt. Außerdem können auch selektionswürdige Merkmale hemmend beeinflussende Gene durch Vermehrung in ihrer Wirkung gesteigert werden. Eine sofort als Folge der Polyploidisierung auftretende Verbesserung der Pflanzen ist also gar nicht zu erwarten. Aus polyploiden Heterozygoten, die gegenüber den diploiden Ausgangspflanzen keine Verbesserung erkennen lassen, können aber durch Selektion unter gewissen Voraussetzungen extrem vorteilhafte Merkmalsbildungen erreicht werden, wie sie auf diploider Basis nicht möglich sind.
\end{abstract}

$\mathrm{V}$ on Hagerup ${ }^{1}$ und Tischler ${ }^{2}$ wurde zuerst wahrscheinlich gemacht, daß die Floren extremer Klimate einen höheren Prozentsatz an polyploiden Arten enthalten als die klimatisch gemäßigter Gebiete. Durch weitere Untersuchungen in den folgenden Jahren wurde das statistische Material, das für eine Häufung von Polyploiden unter harten klimatischen Bedingungen, vor allem in den arktischen Regionen spricht, noch vermehrt. Wesentlich an diesen Zusammenstellungen ist, daß die Ausmerzung durch das Klima, die den erhöhten Prozentsatz an Polyploiden verursachen soll, durch die geringere Artenzahl dieser Floren wirklich nachgewiesen ist. Aus Naturexperimenten und Beobachtungen der Wiederbesiedelung pflanzenfrei gemachter Flächen konnte ebenfalls auf durch-

* „Anpassungsfähigkeit" soll hier in genetisch- phylogenetischem Sinne verstanden sein. K o s t of $\mathrm{f}$ (J. Genet. 36, 447 [1938] und Nature [London] 144, 868 [1939], G y örffy (Planta 32, 15 [1941]) u. a. sind nach ihren experimentellen Beobachtungen zu der Überzeugung gekommen, daß viele Autotetraploide eine größere Anpassungsfähigkeit an Schwankungen der Außenfaktoren auch während der ontogenetischen Entwicklung haben als ihre diploiden Ausgangssippen. schnittlich größere Leistungsfähigkeit der Polyploiden geschlossen werden $\left(\mathrm{Tischler}{ }^{3}\right)$. Aber nicht nur bei der Auslese in der freien Natur, sondern auch bei der künstlichen Auslese, die der Mensch bei der Schaffung seiner Kulturpflanzen betrieb, spielten die Polyploiden diese Rolle: Fast alle wichtigen Kulturpflanzen, auf denen heute die Ernährung der Menschheit beruht, sind Polyploide. Diese Tatsachen verlangen eine genetisch-physiologische Erklärung.

Seitdem auf verschiedene Weise die Polyploidie künstlich ausgelöst werden kann, und vor allem seit der Entdeckung der Colchicinmethode von B l a kes le e und A ver $\mathrm{y}^{4}$, die es gestattet, praktisch aus jeder diploiden leicht eine tetraploide Pflanze herzustellen, ist eine große Zahl von Untersuchungen durchgeführt worden, die den Vergleich morphologischer, anatomischer, physiologischer und ökologischer Merkmale diploider und polyploider Pflan-

1 Hereditas 16, 19 [1932].

2 Botan. Jahrb. 67, 1 [1934], Cytologia, Fujii Jubil. 1, 162 [1937]; Naturwiss. 30, 713 [1942].

3 Z. Naturforschg. 1, 157-159 [1946].

4 J. Heredity 28, 393 [1937]. 
zen zum Gegenstand hatten (z.B. Gy örffy $\mathrm{y}^{5}, \mathrm{Ko}$ st of $f^{6}$, Pirschle $e^{7}$, Schwanitz $z^{8}$, Straub ${ }^{8}$, weitere Literatur in diesen Arbeiten!). Nach all diesen, z. Tl. sehr mühevollen Untersuchungen gilt gemeinsam für alle Polyploiden wohl nur, daß die Zellgröße mit erhöhter Chromosomenzahl ansteigt. Aber das Ausmaß der Vergrößerung ist selbst bei nahen Verwandten schon deutlich sippenspezifisch verschieden (v. Wettstein $\left.{ }^{10}\right)$. Bei den anderen untersuchten Merkmalen läßt sich ein für alle untersuchten Pflanzen einheitliches Bild überhaupt nicht gewinnen. Keinesfalls sind bei künstlich hergestellten Polyploiden gegenüber ihren diploiden Ausgangssippen Merkmalsänderungen, die ihre bessere Eignung in einem Kampf mit ungünstigen Außenfaktoren oder größere, den Pflanzenzüchter interessierende Leistung verständlich machen könnten, auch nur annähernd häufiger als gleichgültige oder gar schädliche Veränderungen. Dieser Widerspruch im Verhalten künstlich hergestellter und in der Natur wild vorkommender oder als Ergebnis jahrzehnte- bis jahrtausendealter Züchtungsarbeit entstandener Polyploider bedarf also der Erklärung. Und tatsächlich ist es möglich, auf Grund weniger Annahmen eine Vorstellung zu entwickeln, die die bessere Anpassungsfähigkeit der Polyploiden verständlich macht und dabei doch die Tatsache berücksichtigt, daß die experimentell frisch hergestellten Polyploiden durchaus nicht in jedem Falle sofort die Verbesserung einer Eigenschaft im Sinne der natürlichen oder künstlichen Auslese erkennen lassen.

Die sofortige Verbesserung der Pflanzen nach Polyploidisierung wäre verständlich, wenn schon dieVervielfachung der Chromosomenzahl, die Kernund Zellvergrößerung an sich, zur Steigerung wertvoller Leistungen führte; das ist nach der oben zitierten Literatur offenbar nicht der Fall. Durch die Verdoppelung der Chromosomenzahl wird aber die Möglichkeit geschaffen, einzelne vorteilhaft wirkende Gene nicht nur wie bei diploiden Pflanzen in zweifacher, sondern bei tetraploiden bis zur vierfachen Menge anzureichern. Sind solche Gene in der diploiden Pflanze vorhanden und steigt ihre Wirkung auch über die Anzahl zwei hinaus an, so können wir bei der tetraploiden eine extremere Ausbildung des vorteilhaften Merkmals er-

5 Der Züchter 12, 130 [1940]; Planta [Berlin] 32, 15 [1941]; Magyar Biol. Kut. Munsk. 362 [1941].

${ }_{6}$ Polyploidie and its role in evolution and plant breeding, Central Agric. Research Inst. Sofia [1941].

Zeitschrift für Naturforschung I, 3 warten als bei der diploiden Pflanze. Es dürfen allerdings im Genom dieser Pflanze dann keine auf die selektionsfördernden Eigenschaften hemmend wirkenden Gene vorhanden sein, deren Wirkungen bei Vermehrung über die Anzahl zwei hinaus in gleichem Maße gesteigert werden. Besonders verwunderlich ist es nicht, wenn diese für die sofortige Verbesserung einer selektionsfördernden Eigenschaft nach Polyploidisierung notwendigen Voraussetzungen nicht eben häufig erfüllt sind. Denn meist dürften mehrere Gene, und zwar die einen in förderndem, andere in hemmendem Sinne, an der Ausbildung jedes Merkmals beteiligt sein, und es ist nicht einzusehen, warum die Wirkung der fördernden Gene mit der Anzahl gesteigert werden soll, die der hemmenden aber nicht oder auch nur weniger. An einem einfachen schematischen Beispiel läßt sich noch leichter zeigen, was gemeint ist. Das Gen $F$ soll die Frostresistenz um $1^{0}$ verbessern und gegenüber $f$ voll dominant sein. Das Gen $H$ hebt die Wirkung von $F$ völlig auf. ff hhPflanzen sollen bei $0^{\circ}$ sterben, $F f h h$ bei $-1^{0}, F F h h$ bei $-2^{0}, F F H h$ bei $-1^{0}$ und $F F H H$ bei $0^{\circ}$. In Tab. 1 sind alle möglichen Kombinationen der Gene $F, f$, $H$ und $h$ in der ersten Reihe, nach Polyploidisierung in der zweiten und nach bestmöglicher Selektion auf Kälteresistenz in der dritten Reihe aufgeschrieben. Hinter jeder Genkombination ist die Temperatur, bei der diese Pflanze voraussetzungsgemäß den Kältetod erleidet, angegeben.

Aus den in Tab. 1 aufgeschriebenen möglichen Kombinationen dieser Gene sieht man, daß in den Fällen I, II, IV, VII und VIII unter der Annahme, daß die Wirkung von $F$ und $H$ mit Vermehrung der Genanzahl über zwei hinaus gleichmäßig steigt, die Kälteresistenz weder durch die Polyploidisierung selbst noch durch nachfolgende Selektion beeinflußt wird. Im Falle III ist ein sofortiger Einfluß der Polyploidisierung vorhanden, der sich durch Selektion nicht vergrößern läßt; in den Fällen V und VI ist ebenfalls schon in der frisch polyploidisierten Pflanze eine Erhöhung der Resistenz im Vergleich mit der Ausgangspflanze zu beobachten, die aber durch Selektion noch gesteigert werden kann. Schließlich haben wir im Falle IX ein Beispiel dafür, daß die Polyploidisierung direkt

7 Planta 32, 517 [1941]; Biol. Zbl. 62, 253 [1942], 62, 455 [1942] ; Z. ind. Abst. Vererbl. 80, 126 [1942] ; 80, 947 [1942]; Planta 32, 517 [1942].

8 Naturwiss. 28, 353 [1940].

9 Biol. Zbl. 60, 659 [1940].

10 Z. ind. Abst. Vererbl. 33, 1 [1924]. 


\begin{tabular}{|c|c|c|c|c|c|}
\hline & I & II & III & IV & $\mathrm{V}$ \\
\hline 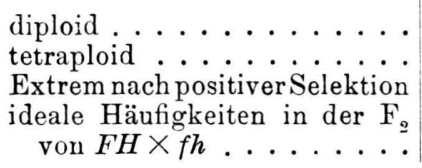 & $\begin{array}{c}F F \quad H H \quad 0^{\circ} \\
F F F F H H H H 0^{\circ} \\
F F F F H H H H 0^{\circ} \\
6,25 \%\end{array}$ & $\begin{array}{l}\text { ff } h h 0^{0} \\
\text { ffff } h h h h 0^{0} \\
\text { ffff } h h h h 0^{\circ} \\
\quad 6,25 \%\end{array}$ & $\begin{array}{c}F F \quad h h-2^{\circ} \\
F F F F h h h-4^{0} \\
F F F F h h h h-4^{\circ} \\
6,25 \%\end{array}$ & $\begin{array}{l}\text { ff } \begin{array}{c}H H \\
\text { ffff } 0^{\circ} \\
\text { ffff } H H H H 0^{\circ}\end{array} \\
6,25 \%\end{array}$ & $\begin{array}{c}F f \quad h h-1^{\circ} \\
F F f f h h h h-2^{\circ} \\
F F F F h h h h-4^{0} \\
12,5 \%\end{array}$ \\
\hline
\end{tabular}

\begin{tabular}{|c|c|c|c|c|}
\hline & VI & VII & VIII & IX \\
\hline 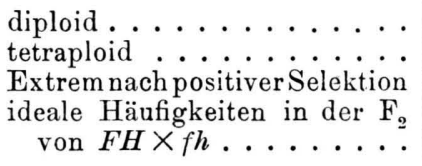 & $\begin{array}{c}F F \quad H h-1^{0} \\
F F F F H H h h-2^{\circ} \\
F F F F \text { hhhh }-4^{\circ} \\
12,5 \%\end{array}$ & 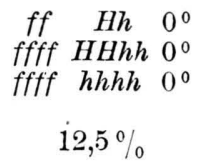 & 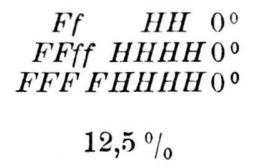 & 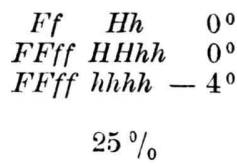 \\
\hline
\end{tabular}

Tab. 1.

gar keinen Einfluß auf die Resistenz hat, eine Selektion in den folgenden Generationen aber sehr wohl zu einem in diploiden Pflanzen unerreichbaren Extrem von Kälteresistenz führen kann.

In diesem Schema war angenommen, daß $H$ die Wirkung von $F$ aufhebt, selbst aber keinen Einfluß auf die Kälteresistenz hat. Es ist auch denkbar, daß $H$ die Wirkung von $F$ aufhebt und selbst die Kälteresistenz negativ beeinflußt. Unter dieser Annahme würde Fall VIII folgendermaßen aussehen:

$\begin{array}{lccr}\text { diploid } & F f & H H & +1^{\circ} \\ \text { tetraploid } & F F f f & H H H H & +2^{\circ} \\ \text { Extrem nach } & & & \\ \text { positiver Selektion } & \text { FFFF } & \mathrm{HHHH} & 0^{\circ}\end{array}$

In diesem Falle ist es also möglich, daß die Polyploidisierung selbst zunächst eine Verschlechterung gegenüber der diploiden Ausgangspflanze bewirkt, durch Selektion aber nicht nur eine Aufhebung dieser Verschlechterung, sondern sogar eine Verbesserung über den Anfangszustand hinaus erzielt werden kann.

Das schematische Beispiel ließe sich noch in verschiedener Weise abändern, darauf kann aber verzichtet werden, da das Grundsätzliche genügend deutlich gemacht sein dürfte. Den idealen Häufigkeiten der einzelnen Klassen, wie sie in der $\mathrm{F}_{2}$ einer Kreuzung $F H \times f h$ und nachfolgender Polyploidisierung auftreten würden (unterste Reihe der Tab. 1!), darf kein zu großer Wert beigelegt werden. Immerhin sieht man, daß selbst bei so günstigen Annahmen $43,75 \%$ der Individuen der $\mathrm{F}_{\mathbf{2}}$ nach Polyploidisierung gar keinen Erfolg versprechen; $6,25 \%$ haben nach Polyploidisierung eine erhöhte Kälteresistenz, die durch Selektion nicht verbessert werden kann;
25\% ergeben eine kleine Verbesserung direkt nach Polyploidisierung mit der Möglichkeit, durch Selektion das Extrem zu erreichen, und $25 \%$ zeigen zunächst keinen Erfolg der Polyploidisierung, lassen sich aber durch Selektion bis zum Extrem verbessern.

Daß in praxi andere Störungen die Erfolgswahrscheinlichkeit ganz wesentlich einschränken werden, bedarf keines besonderen Hinweises. Es sei nur an die nach Polyploidisierung geradezu unvermeidlichen Fertilitätsherabsetzungen und die für das gewählte Beispiel besonders schwerwiegende Zunahme des Zellvolumens und den herabgesetzten osmotischen Wert des Zellsaftes erinnert!

Zur Erklärung der besseren Anpassungsfähigkeit der Polyploiden in der Natur und als Ergebnis der Kulturpflanzenzüchtung brauchen wir demnach nur eine Annahme, nämlich, daßes selektionswürdige Merkmale beeinflussende Gene gibt, deren Wirkung mit der Menge auch über die Anzahl zwei hinaus noch gesteigert werden kann.

Die Tatsache, daß die experimentell frisch hergestellten Polyploiden nur selten die Verbesserung selektionswürdiger Merlimale sofort erkennen lassen, ist leicht unter folgenden Annahmen zu verstehen: 1. können in einem gegebenen Genom Gene für selektionswürdige Merkmale, die nicht schon in einfacher oder zweifacher Menge ihr Wirkungsmaximum erreichen, fehlen. 2. kann auch beim Vorhandensein solcher Gene die Anwesenheit von anderen Genen, die auf die Ausbildung des interessierenden Merkmals hemmend wirken, und deren Wirkung ebenfalls bei Vermehrung über die zweifache Quantität ansteigt, eine günstige Wirkung 
der Polyploidisierung verhindern. 3. wird die regelmäßig nach Erhöhung der Genomquantität mehr oder weniger ausgeprägte Vergrößerung des Zellvolumens und die damit manchmal verbundenen Monstrositäten der Organgestaltung und Abweichungen des Zellstoff wechsels, z. B. gesenkter osmotischer Wert des Zellsaftes (Becker ${ }^{11}$ ), auch etwaige kleine, besondere Merkmale betreffende Verbesserungen zunächst überdecken.

Es fragt sich nun, ob die gemachten Annahmen nach unseren bisherigen Kenntnissen berechtigt sind. Schon aus den Arbeiten von F.v. Wettste in (1924) ist bekannt, daß die Wirkung mancher Gene weiter ansteigt, wenn man ihre Quantität über das zweifache hinaus steigert. Das Gen $B$ beeinflußt z. B. die Form des Deckels der Sporogonkapsel bei Funaria hygrometrica. Je mehr B-Gene vorhanden sind, um so größer wird der Quotient Durchmesser : Höhe der Deckelkapsel, wie folgende Übersicht zeigt:

\begin{tabular}{c|c|c|c|c|c}
\hline $\begin{array}{c}\text { Genetische } \\
\text { Konstitution }\end{array}$ & $b b b b$ & $B b b b$ & $B B b b$ & $B B B b$ & $B B B B$ \\
\hline $\begin{array}{c}\text { Durchmesser: Höhe } \\
\text { der Deckelkapsel }\end{array}$ & 2,68 & $\mathbf{2 , 5 9}$ & $\begin{array}{l}3,04^{12} \\
\mathbf{2 , 7 5 ^ { 1 2 }}\end{array}$ & 5,13 & 5,29 \\
\hline
\end{tabular}

Noch besser zeigt sich - ohne die Störung bei den $B b b b$-Pflanzen! - die Abhängigkeit der Merkmalsausbildung von der nullfachen bis vierfachen Menge des Gens $B$ bei der in Tafel II, Fig. 14-17, in der Arbeit von v. Wettstein wiedergegebenen „Neigung der Kapsel“".

Schöne Beispiele finden sich auch in einer Arbeit über die Genetik und Biochemie der Blütenfarben tetrasomisch spaltender Gartenhybriden von Dahlia variabilis von $\mathrm{L}$ a w r e $\mathrm{n}$ c e und $\mathrm{S} \mathrm{c}$ o t $\mathrm{t}-\mathrm{M}$ o n crieff ${ }^{13}$. Die Wirkung des Gens $A$ (Ausbildung heller Anthocyanfarbstoffe auslösend) steigt von der einfachen bis zur vierfachen Menge gleichmäßig an.

Auch für die erste Annahme, die uns das Ausbleiben positiver Effekte sofort nach Polyploidisierung verständlich macht, findet man in dieser Arbeit Modelle: Die Gene $B$ (Ausbildung tiefer Anthocyanfarbstoffe auslösend) und $Y$ (Ausbildung gelber Flavone auslösend), sind schon in einfacher Menge voll dominant, ihre Wirkung nimmt mit

11 Z. ind. Abst. Vererbl. 60, 17 [1931].

12 Oberer Wert aus der Kreuzung $B B \times b b$, unterer aus $B b \times B b$.
Steigerung ihrer Menge auf das zwei- bis vierfache nicht mehr zu. Besonders interessant verhält sich das Gen I (Ausbildung elfenbeinfarbener Flavone auslösend). In einfacher Menge entfaltet es eine kaum wahrnehmbare Wirkung. Das Wirkungsmaximum ist aber schon bei zweifacher Genquantität erreicht. Die drei- oder vierfache Menge dieses Gens bringt keine Steigerung der Wirkung mehr hervor.

Aus der Arbeit von v. Wettstein läßt sich für ein begrenztes Ansteigen den Genwirkung mit der Genmenge das Gen $C$, welches die Kapselfärbung von Funaria hygrometrica kontrolliert, anführen.

\begin{tabular}{|c|c|c|c|c|c|}
\hline $\begin{array}{c}\text { Genetische } \\
\text { Kon- } \\
\text { stitution }\end{array}$ & $\operatorname{cccc}$ & Cecc & $C C_{c} c$ & $C C C c$ & $C C C C$ \\
\hline $\begin{array}{c}\text { Färbung } \\
\text { der } \\
\text { Kapsel }\end{array}$ & $\begin{array}{l}\text { rost- } \\
\text { ocker }\end{array}$ & $\begin{array}{l}\text { rost- } \\
\text { ocker }\end{array}$ & $\begin{array}{l}\text { gelb- } \\
\text { orange }\end{array}$ & $\begin{array}{c}\text { orange bis } \\
\text { schwarz- } \\
\text { rot }\end{array}$ & $\begin{array}{c}\text { orange bis } \\
\text { schwarz- } \\
\text { rot }\end{array}$ \\
\hline $\begin{array}{c}\text { Färbung } \\
\text { des } \\
\text { Deckels }\end{array}$ & ocker & ocker & $\begin{array}{c}\text { schwarz- } \\
\text { rot }\end{array}$ & $\begin{array}{c}\text { schwarz- } \\
\text { rot }\end{array}$ & $\begin{array}{l}\text { schwarz- } \\
\text { rot }\end{array}$ \\
\hline
\end{tabular}

Hier ist für die Deckelfärbung das Maximum der Wirkung des Gens $C$ schon bei der zweifachen, das für die Kapselfärbung bei der dreifachen Genmenge erreicht. Das Beispiel zeigt auch, wie bei Polyploiden qualitativ neue Merkmale, wenn man die schwarzrote Färbung als etwas qualitativ Neues auffassen will, auftreten können. In diploiden Sporogonen ist der tiefste Farbton orange.

\begin{tabular}{c|c|c|c}
\hline $\begin{array}{c}\text { Genetische } \\
\text { Konstitution }\end{array}$ & $c c$ & $C c$ & $C C$ \\
\hline Färbung der Kapsel & ocker & gelborange & orange \\
\hline Färbung des Deckels & ocker & ocker & orange
\end{tabular}

Auch die Verschiebung der Farbtiefe zwischen Kapsel und Deckel ist Folge der Polyploidie. In Diploiden ist der Deckel eher heller gefärbt als die Kapsel, in Tetraploiden von der zweifachen $C$ Menge an ist die Deckelfärbung tiefer als die Kapselfärbung.

Für die zweite Annahme kann man das von Law rence analysierte Gen $H$ (Gegenspieler von $Y$, die Ausbildung gelber Flavone hemmend) an-

13 J. Genet. 30, 155 [1935]. 
führen. Seine Wirkung steigt kumulativ von der einfachen bis zur vierfachen Menge.

Schließlich drittens die allgemeinen Wirkungen der vermehrten Genomquantität auf die Zellgröße und deren direkte und indirekte Folgen! Die Tatsache des Gigaswuchses und seiner Auswirkungen sind zu bekannt, und die Erscheinung so verbreitet, daß sich die Anführung besonderer Beispiele erübrigt.

Somit erscheinen unsere Annahmen ausreichend plausibel zu sein und die auf ihnen beruhende Vorstellung dürfte grundsätzlich das Richtige treffen. Nach dieser Vorstellung muß Polyploidie nicht unbedingt für jede Pflanze einen Vorteil vor der Diploidie haben. Es sind Genome denkbar, bei denen eine Vervielfachung nicht zur Verbesserung selektionswürdiger Merkmale führt. Es ist ja auch keineswegs so, daß in extremen Klimaten nur polyploide Arten vorkämen, und es gibt auch unter den bedeutenden Kulturpflanzen diploide, wie z. B. die Gerste, die wohl das älteste unserer Kulturgetreide überhaupt ist. In solchen Fällen dürfte sich die Diploidie weniger deshalb erhalten haben, weil Polyploidie spontan zu selten auftritt, als weil die Genome dieser Pflanzen auch in allen bisher aufgetretenen Abwandlungen nach Polyploidisierung keine Verbesserung selektionsw ürdiger Merkmale ergeben haben.

Wenn die Beispiele, die unsere Annahme plausibel machen, aus der Literatur auch noch zu vermehren wären, so soll doch nicht übersehen werden, daß die experimentelle Arbeit auf dem Polyploidiegebiet bisher nur sehr selten unter diesen Gesichtspunkten betrieben wurde. Untersuchungen an experimentell hergestellten Polyploiden, bei denen die quantitative Wirkung einzelner Gene bei sonst konstantem Genom untersucht wird, würden immer noch als Sicherung für unsere Vorstellungen wünschenswert sein. Dagegen ist die Anhäufung von weiterem Material, welches dem Vergleich diploider und experimentell hergestellter polyploider Pflanzen dient, und wie es in den oben zitierten zusammenfassenden Darstellungen reichlich enthalten ist, nicht mehr notwendig.

Auf die Frage, warum die einfache genphysiologische Vorstellung zur Erklärung der besseren

14 In einem Vortrag „Genetik und Evolution“ auf der Tagung der Dtsch. Ges. f. Vererbungswiss. in Würzburg 1938 hat der Verf. diese Vorstellung zwar schon angeführt und auf der Tagung der Dtsch. Bot. Ges. 1942 in Würzburg nach dem Vortrag von R. Griesinger (Ber. dtsch. bot. Ges. 60, 36 [1942]) auch mit
Anpassungsfähigkeit der Polyploiden nicht schon früher in eindeutiger Weise formuliert wurde ${ }^{14}$, sind mehrere Antworten möglich. Einem Teil der Genetiker dürfte sie so selbstverständlich sein, daß sie sich bisher mit kurzen Andeutungen des Grundgedankens begnügt haben. Andere, vorwiegend pflanzengeographisch, physiologisch oder züchterisch interessierte Untersucher haben aber anscheinend bei dem Versuch, die Erklärung für das Verhalten der Polyploiden in der Natur und auf dem Gebiet der Kulturpflanzen aus messenden Vergleichen diploider und experimentell hergestellter polyploider Pflanzen zu finden, direkte Erfolge erwartet und sind durch die verwirrenden Ergebnisse von einer einheitlichen Deutung abgekommen. Aus manchen Arbeiten glaubt man auch die Hoffnung auf die Entdeckung irgendeines neuen Prinzips, das zur Aufklärung der Diskrepanz zwischen dem Verhalten natürlicher und frisch hergestellter $\mathrm{Po}_{-}$ lyploider nötig sein soll, heraushören zu können. Solche Autoren dürften von der geńphysiologischen Deutung, die nur Bekanntes an Annahmen verwendet, enttäuscht sein.

Für die züchterische Praxis ist eine zutreffende Vorstellung von der Natur der Anpassungsfähigkeit der Polyploiden als Richtschnur für die großen Aufwand an Material und lange Zeit erfordernden Arbeiten nicht unwesentlich. Nach einer übertrieben großen Hoffnungsfreudigkeit, die in den Kreisen der Züchter nach Entdeckung des Colchicinverfahrens zu beobachten war, kam die große Enttäuschung nach Bekanntwerden der Leistungsvergleiche diploider und experimentell hergestellter polyploider Pflanzen. Gelegentlich hört man auch, die Ergebnisse der Grundlagenforschung seien noch nicht genügend einheitlich und gesichert, um sie für die Praxis verwerten zu können. Das ist aber, wie wir gesehen haben, nicht der Fall. Auf Grund unserer heutigen Kenntnisse und der genphysiologischen Vorstellung von der besseren Anpassungsfähigkeit der Polyploiden lassen sich für die Praxis folgende Richtlinien für die Polyploidiezüchtung geben: 1 . Als Objekte sind alte Kulturpflanzen, wie z. B. die Gerste, ungeeignet. Wenn im Genom solcher Pflanzen für die Polyploidiezüchtung geeignete Kombinationen möglich gewesen wären, sollten die Polyploiden im Laufe der jahr-

mehreren Kollegen öfentlich diskutiert. Die gedruckten Publikationen (Z. ind. Abst. Vererbl. 76, 249 ff. [1939]; Ber. dtsch. bot. Ges. 60, 13 [1942]) sind aber so kurz gefaßt, daß die Allgemeinverständlichkeit offenbar gelitten hat. 
tausendelangen Züchtungsarbeit auch entstanden sein. Als Objekte kommen also vorwiegend neue, züchterisch noch wenig bearbeitete Pflanzen in Frage. 2. Es ist falsch, nur wenige und womöglich noch genetisch einheitliche Linien zu polyploidisieren. Es sollten möglichst viele Linien oder besser in möglichst vielen Genen heterozygote Pflanzen polyploidisiert werden. 3. Der Erfolg ist bei der Polyploidiezüchtung nicht etwa früher als bei Züchtungsarbeiten an diploiden Pflanzen, sondern später zu erwarten. Denn das Herausspalten der günstigen Kombinationen erfolgt auf polyploider Basis selbstverständlich noch langsamer als auf diploider. Die so umfangreich wie möglich aufzuziehenden Nachkommenschaften müssen also viele Generationen durchselektioniert werden. Wenn es sich um Resistenzzüchtung handelt, muß noch mehr Sorgfalt darauf verwandt werden als bei Diploiden, die noch nicht widerstandsfähigen Heterozygoten zu erhalten. Wenn so scharfe Selektionsbedingungen angewendet werden, daß große Mengen der Versuchspflanzen zugrundegehen, wird es sich empfehlen, in jeder Generation nur etwa die Hälfte des Materials diesen Bedingungen zu unterwerfen und die nächste Generation nicht nur aus den Überlebenden des Versuchs, sondern zunächst auch aus dem nicht selektionierten Anteil aufzubauen.

Besondere Beachtung verdient - aber doch wohl nur scheinbar - der durch vergrößertes Zellvolumen verursachte Gigaswuchs der Polyploiden. Diese Eigenschaft scheint bei oberflächlicher Betrachtung die Herstellung von Polyploiden vom züchterischen Standpunkt aus wünschenswert zu machen. Die Ertrags- und Leistungsprüfungen von experimentell hergestellten Polyploiden zeigen aber, daß diese Folgen der Polyploidie, zumal mit ihnen meist geringere Wachstumsgeschwindigkeit und herabgesetzte Fertilität verbunden sind, eher schädlich als nützlich sind. Abgesehen sei hier von der Entstehung allopolyploider, fertiler Stämme aus sterilen Artbastarden in der Natur oder ihrer Herstellung für züchterische Zwecke. Ihre Bedeutung ist für die Evolution sicher erwiesen ( $M$ ü n tz in $g^{15}$, v. Wettste i ${ }^{16}$ ), und in der Pflanzenzüchtung können sie entweder direkt wegen der in ihnen für immer fixierten Heterosis der $\mathrm{F}_{1}$-Bastarde oder als Ausgangspflanzen für weitere Kreuzungsarbeit

15 Hereditas 14, 153 [1930]; 16, 105 [1932].

16 Naturwiss. 20, 981 [1932]. wichtig sein. Die sicherlich nicht auf Artbastardierung zurückgehenden natürlichen Polyploiden zeigen die Gigaseigenschaften meist nicht in solchem Ausmaße wie die frisch hergestellten und vor allem fehlen ihnen die Wachstumshemmungen, Monstrositäten und Fertilitätsstörungen. Auf diese Tatsache hat v. Wettste in in seinen neueren Arbeiten $^{17}$ mit besonderem Nachdruck hingewiesen und auch durch Beobachtungen an dem künstlich durch Polyploidisierung aus Bryum caespititium hergestellten Bryum Corrensii gezeigt, daß ursprünglich vorhandene Gigasmerkmale und die damit verbundenen Störungen der. Fertilität in verhältnismäßig kurzer Zeit verschwinden können. Bei Berücksichtigung der Tatsache, daß auch das Ausmaß der nach Polyploidisierung eintretenden Zellvergrößerung von mendelnden Genen abhängig ist (v. Wettstein 1924, M. Tobler ${ }^{13}$ ) ist aber auch dieses Phänomen auf selektionistischer Basis verständlich. v. Wettstein hat selbst den Fall seines Bryum Corrensii in seiner zweiten diesbezüglichen Mitteilung (v. Wettstein u.Stra $u b^{19}$ ) in diesem Sinne zu deuten versucht, wobei allerdings die Annahme gemacht werden mußte, daß ein die Zellgröße herabsetzendes Gen während der Beobachtungszeit durch Mutation entstanden ist. Geht die Polyploidisierung von stark heterozygoten Pflanzen aus, so können - ganz dem schematischen Beispiel für Frostresistenz entsprechend - auch Gene, die die Zellgröße beeinflussen, günstige Möglichkeilen für die Selektion bieten; und dabei brauchen sie sich in den experimentell hergestellten Polyploiden durchaus nicht gleich auszuwirken. Somit wird also auch das scheinbar ein Sonderproblem enthaltende Kapitel des Gigaswuches frisch hergestellter Polyploider und das Verschwinden dieser Eigenschaft in der Natur von der allgemeinen genphysiologischen Vorstellung aus, wie wir sie auf Grund der heute bekannten Tatsachen entwickeln konnten, grundsätzlich lösbar. Es soll keineswegs verkannt werden, daß weitere Beobachtungen auf experimenteller Grundlage gerade zu dieser Frage als Stützen oder gegebenenfalls als Ansätze für Erweiterungen oder gar als Korrekturen unserer Vorstellung wünschenswert sind.

17 Ber. dtsch. bot. Ges. 58, 344 [1940]; F. v. Woess, Z. ind. Abst. Vererbl. 79, 444 [1941].

18 Z. ind. Abst. Vererbl. 60, 39 [1931].

19 Z. ind. Abst. Vererbl. 80, 271 [1942]. 